\title{
Treatment of borderline personality disorder and co-occurring anxiety disorders
}

\author{
Melanie S. Harned* and Helen R. Valenstein
}

\author{
Address: Behavioral Research and Therapy Clinics, Department of Psychology, Box 355915, University of Washington, Seattle, WA, 98195, USA \\ *Corresponding author: Melanie S. Harned (mharned@uw.edu) \\ Fl000Prime Reports 2013, 5:15 (doi:10.12703/P5-15) \\ This is an open-access article distributed under the terms of the Creative Commons Attribution-Non Commercial License \\ (http://creativecommons.org/licenses/by-nc/3.0/legalcode), which permits unrestricted use, distribution, and reproduction in any medium, \\ provided the original work is properly cited. You may not use this work for commercial purposes. \\ The electronic version of this article is the complete one and can be found at: http://f $1000 . c o m / p r i m e /$ reports/m/5/I5
}

\begin{abstract}
Anxiety disorders are highly prevalent among individuals with borderline personality disorder, with comorbidity rates of up to $90 \%$. Anxiety disorders have been found to reduce the likelihood of achieving remission from borderline personality disorder over time and to increase the risk of suicide and self-injury in this population. Evidence-based treatments for borderline personality disorder have not sufficiently focused on targeting anxiety disorders, and their effects on these disorders are either limited or unknown. Conversely, evidence-based treatments for anxiety disorders typically exclude suicidal, self-injuring, and seriously comorbid patients, thereby limiting their generalizability to individuals with borderline personality disorder. To address these limitations, recent research has begun to emerge focused on developing and evaluating treatments for individuals with co-occurring borderline personality disorder and anxiety disorders, specifically posttraumatic stress disorder (PTSD), with promising initial results. However, there is a need for additional research in this area, particularly studies evaluating the treatment of anxiety disorders among high-risk and complex borderline personality disorder patients.
\end{abstract}

\section{Introduction}

Borderline personality disorder is a severe and complex psychological disorder characterized by long-term patterns of intense emotions, impulsive and self-destructive behaviors, and chaotic relationships. Borderline personality disorder is also associated with high rates of comorbidity. Individuals with borderline personality disorder meet criteria for an average of 3.0 to 3.4 current Axis I disorders (e.g. mood, anxiety, eating, psychotic, and substance use disorders) and 4.2 to 4.8 lifetime Axis I disorders [1,2]. Although emphasis is often placed on the comorbidity between borderline personality disorder and mood disorders, anxiety disorders are equally and highly prevalent, with approximately $75-90 \%$ of individuals with borderline personality disorder meeting criteria for at least one lifetime anxiety disorder [3-5]. Despite the negative prognostic significance of anxiety disorders on borderline personality disorder [6], there is a paucity of research examining effective treatments for these frequently co-occurring disorders, particularly among severe borderline personality disorder patients. The present review will describe the existing research in this area and make suggestions for future directions.

The prevalence, course, and impact of anxiety disorders in borderline personality disorder Although the high rate of comorbidity between borderline personality disorder and PTSD has received the most theoretical and empirical attention, each of the anxiety disorders has been found to be prevalent among individuals with borderline personality disorder. Among borderline personality disorder inpatients, $88 \%$ meet criteria for a lifetime anxiety disorder, including PTSD (56\%), panic disorder $(48 \%)$, social anxiety disorder $(46 \%)$, specific phobia (32\%), obsessive compulsive disorder (16\%), generalized anxiety disorder (14\%), and agoraphobia $(12 \%)$ [4]. Similarly, high rates of current and lifetime anxiety disorders have been found among borderline personality disorder outpatients [1], treatment-seeking individuals with borderline personality disorder [7], and 
community samples of borderline personality disorder individuals [5]. Anxiety disorders are more prevalent among borderline personality disorder patients than other clinical populations $[1,4]$, and among women than men with borderline personality disorder $[5,8]$. Anxiety disorders also have a complex and variable course in borderline personality disorder, with high rates of remission $(77-100 \%)$, recurrence (30-65\%), and new onsets (15-45\%) over 10 years of prospective follow-up $[9,10]$. Perhaps most critically, anxiety disorders are associated with a heightened risk of suicidal and non-suicidal selfinjury among individuals with borderline personality disorder [11-18] and have been found to decrease the likelihood of achieving remission from borderline personality disorder over time [6].

\section{The efficacy of borderline personality disorder treatments for co-occurring anxiety disorders}

To date, there have been 14 randomized controlled trials (RCTs) that have examined the effects of borderline personality disorder treatments on anxiety, with all but one of these focused on general anxiety severity as opposed to specific anxiety disorder diagnoses (Table 1). The one study that has evaluated the effects of borderline personality disorder treatment on anxiety disorder diagnoses found that, among suicidal borderline personality disorder women in Dialectical Behavior Therapy, rates of remission from anxiety disorders ranged from $35-47 \%$ [2]. These remission rates did not differ from those found in the Community Treatment by Experts control condition (24-54\%), and were lower than those found for mood, substance, and eating disorders in Dialectical Behavior Therapy (64-88\%) [2]. The remaining 13 studies have examined general anxiety severity and have found mixed results. At post-treatment and/or follow-up, nine studies found significant decreases in anxiety [19-28], one study did not find a significant decrease [29], and three studies did not report prepost changes [30-33]. Additionally, six studies found significant treatment differences in anxiety outcomes $[19-22,26,27,33]$, whereas seven studies found no differences between treatments [23-25,28-32]. Although these studies generally indicate that treatment for borderline personality disorder is associated with a significant reduction in anxiety severity, it is unknown if or how these improvements are related to anxiety disorder diagnostic status. Of note, several borderline personality disorder treatments that have been examined in RCTs have not yet been evaluated in terms of their impact on anxiety outcomes, including Schema-Focused Therapy, Systems Training for Emotional Predictability and Problem Solving, Dynamic Deconstructive Psychotherapy, and General Psychiatric Management (see [34] for a review of these treatments).
The efficacy of anxiety disorder treatments for individuals with borderline personality disorder Although numerous evidence-based treatments for anxiety disorders are available, borderline personality disorder patients, particularly those with a severe level of disorder, are likely to be excluded from these treatments due to suicidality, self-injurious behavior, and other co-occurring problems that are deemed primary or in need of immediate treatment $[35,36]$. As a result, few studies have evaluated the efficacy of anxiety disorder treatments among individuals with borderline personality disorder, and the available research is limited to PTSD treatment studies. Results from three RCTs of cognitive-behavioral treatments for PTSD indicate that patients with borderline personality disorder or borderline personality characteristics exhibited a comparable rate of improvement as patients without borderline personality disorder or borderline personality characteristics [37-39]. However, borderline personality disorder patients $(11 \%)$ were less likely than those without borderline personality disorder (51\%) to achieve good end-state functioning [37]. Importantly, each of these studies excluded patients with acute suicidality as well as one or more other problems common in severe borderline personality disorder (e.g. substance use disorder, recent self-injury). Although some research has evaluated the impact of personality disorders on treatment outcome for nonPTSD anxiety disorders $[40,41]$, no studies have specifically evaluated the effect of borderline personality disorder. Thus, research on the use of anxiety disorder treatments among individuals with borderline personality disorder is limited by the small number of studies available, the exclusion of severe borderline personality disorder patients, and the lack of studies on anxiety disorders other than PTSD.

\section{Recent advances in psychosocial treatments for borderline personality disorder and co-occurring anxiety disorders}

Given the paucity of empirical data on the treatment of co-occurring borderline personality disorder and anxiety disorders, recent research has begun to emerge focused on developing and evaluating treatments for this population. To date, this research has focused exclusively on individuals with comorbid borderline personality disorder and PTSD. Harned and colleagues have developed a one-year outpatient treatment for suicidal and self-injuring individuals with borderline personality disorder and PTSD that integrates Dialectical Behavior Therapy with a modified version of Prolonged Exposure therapy for PTSD $[42,43]$. To date, this treatment has been evaluated in an open trial $(n=13)$ with results indicating that it is feasible and safe to administer, and is associated with large and significant improvements in 


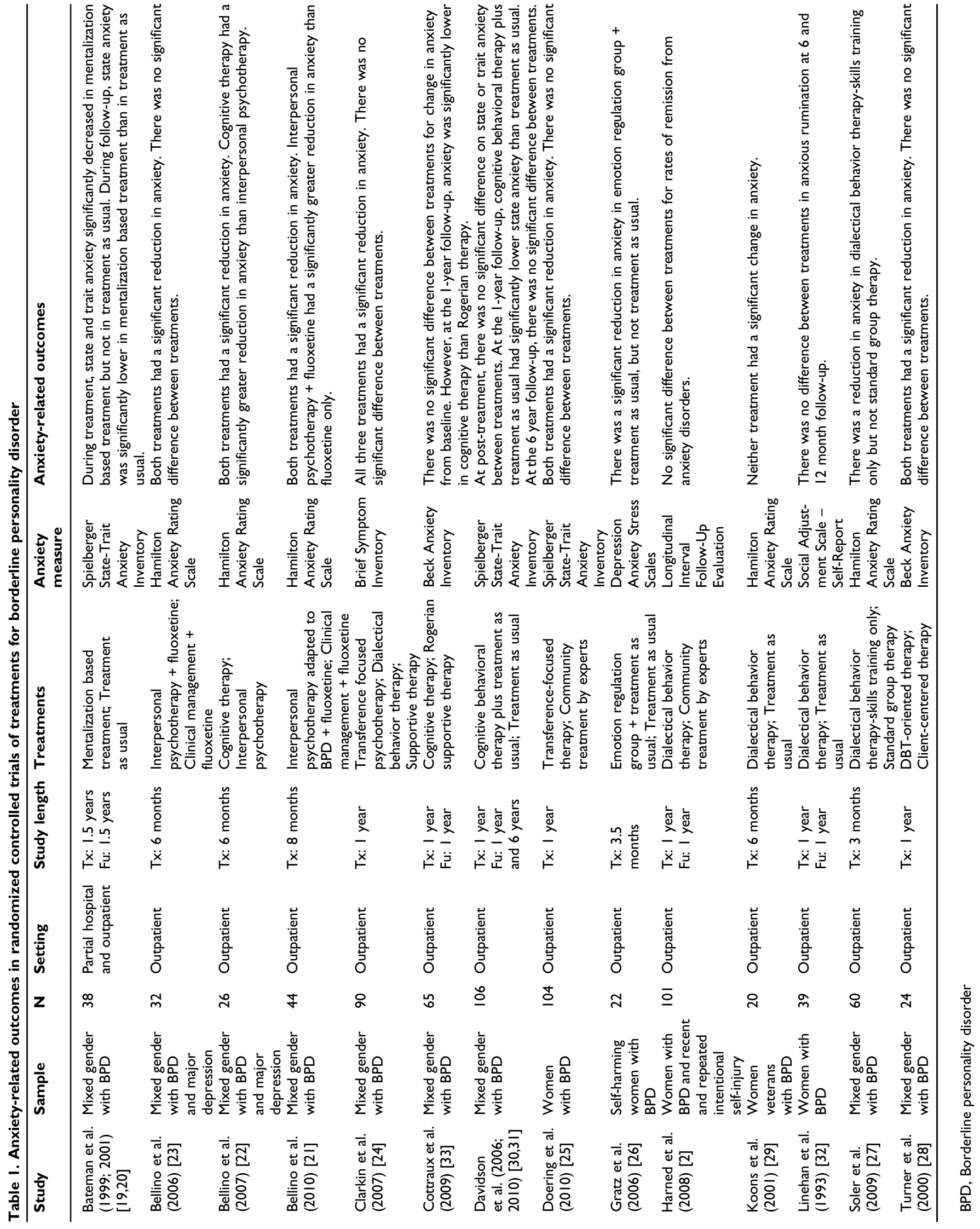


PTSD (pre-post $d=1.4-1.7$; remission rate $=60-72 \%$ ), intentional self-injury, and a number of secondary outcomes [44]. Pabst and colleagues [45] conducted a feasibility trial of Narrative Exposure Therapy for women with borderline personality disorder and PTSD $(n=10)$. Treatment primarily occurred in an inpatient unit, lasted an average of 14 sessions, and excluded patients who were unwilling to consent to a no-suicide contract, had attempted suicide in the past eight weeks, or had other severe comorbidities. The treatment was associated with significant reductions in PTSD $(g=0.92)$ and several secondary outcomes. Finally, although not intended specifically for borderline personality disorder patients, Bohus and colleagues have developed a 12-week residential treatment for women with childhood sexual abuse-related PTSD plus a current diagnosis of major depression, eating disorder, substance use disorder, and/ or at least four borderline personality disorder criteria. Individuals with a suicide attempt in the past four months are excluded. This treatment combines modified Dialectical Behavior Therapy and trauma-focused cognitivebehavioral approaches [46] and has been evaluated in an open trial $(n=29)$ [47] and an RCT $(n=74)$ [48], in which borderline personality disorder patients constituted $24-42 \%$ of the samples. Both studies have shown large and significant reductions in PTSD $(d=1.2-1.5$; remission rate $=35-36 \%$ ) and a number of secondary outcomes $[47,48]$ with one study finding that results were comparable between patients with and without borderline personality disorder [48]. Although these treatments have shown promising results, more research is needed to replicate these findings using randomized controlled designs with larger samples of borderline personality disorder patients.

\section{Future directions}

Given the high rate of co-occurring anxiety disorders among borderline personality disorder patients, as well as the negative impact of these disorders on achieving remission from borderline personality disorder, it is imperative that additional research continues to evaluate how to effectively treat anxiety disorders among individuals with borderline personality disorder. To date, the available research has focused exclusively on evaluating treatments for PTSD among borderline personality disorder patients, and no research has yet evaluated treatments for other types of anxiety disorders in borderline personality disorder. In addition, with the exception of Dialectical Behavior Therapy, the impact of existing borderline personality disorder treatments on specific anxiety disorders has not been examined.

As efforts to develop and evaluate treatments for cooccurring borderline personality disorder and anxiety disorders continue, attention should be paid to tailoring these treatments to borderline personality disorder patients with various levels of disorder. For example, it may be the case that existing, brief ( $9-12$ week) anxiety disorder treatments can be implemented safely and effectively among borderline personality disorder patients with a mild level of disorder (e.g. those without suicidal and self-injurious behaviors or severe comorbidities) $[37,38]$. Borderline personality disorder patients with a moderate level of disorder (e.g. low risk non-suicidal selfinjury without serious suicidality, significant but nondisabling deficits in interpersonal and emotion regulation skills) may benefit from longer (12-16 week) and/or more intensive (e.g. residential) sequential treatments that implement strategies from borderline personality disorder treatments (e.g. skills training) prior to targeting anxiety disorders [47-49]. Finally, longer-term treatment (e.g. one year) that provides integrated treatment for borderline personality disorder and anxiety disorders may be necessary for borderline personality disorder patients with a severe level of disorder (e.g. recent serious suicidal and/ or self-injurious behaviors, severe comorbidities, disabling psychosocial impairment) [44].

Within this general research agenda, several important questions remain to be addressed. First, it will be important to develop empirically-derived criteria for determining when to target anxiety disorders among individuals with borderline personality disorder, particularly in the context of multiple other severe problems. For example, it is not yet clear whether anxiety disorders can be safely and effectively treated among borderline personality disorder patients who are actively engaging in non-suicidal self-injury. It is also possible that these readiness criteria may differ depending on the treatment setting (e.g. inpatient/ residential versus outpatient), type of anxiety disorder (e.g. PTSD versus other diagnoses), and/or the relationship of anxiety disorder symptoms to suicidal and self-injurious behaviors. Finally, some research has begun to identify common factors underlying borderline personality disorder and anxiety disorders, including a tendency to experience negative emotions combined with efforts to try to alter or avoid these emotions [50-53]. The extant research suggests that borderline personality disorder treatments that address these common factors may be sufficient to facilitate improvements in general anxiety severity. However, additional research is needed to determine whether more targeted anxiety disorder treatment is necessary to achieve full remission from these disorders during treatment for borderline personality disorder.

\section{Abbreviations}

PTSD, posttraumatic stress disorder; RCT, randomized controlled trial. 


\section{Disclosures}

Melanie Harned is a Dialectical Behavior Therapy trainer and consultant.

\section{Acknowledgments}

Dr. Harned is supported by a grant from the National Institute of Mental Health (R34MH082143).

\section{References}

I. Zimmerman M, Mattia Jl: Axis I diagnostic comorbidity and borderline personality disorder. Compr Psychiatry 1999, 40: 245-52.

\section{FIOOOPRIM
RECOMMENDED}

2. Harned MS, Chapman AL, Dexter-Mazza ET, Murray A, Comtois KA, Linehan MM: Treating co-occurring Axis I disorders in recurrently suicidal women with borderline personality disorder: a 2-year randomized trial of dialectical behavior therapy versus community treatment by experts. J Consult Clin Psychol 2008, 76:1068-75.

3. Linehan MM, Comtois KA, Murray AM, Brown MZ, Gallop RJ, Heard HL, Korslund KE, Tutek DA, Reynolds SK, Lindenboim N: Two-year randomized controlled trial and follow-up of dialectical behavior therapy vs therapy by experts for suicidal behaviors and borderline personality disorder. Arch Gen Psychiatry 2006, 63:757-66.

\section{FlOOOPrime}

\section{RECOMMENDED}

4. Zanarini MC, Frankenburg FR, Dubo ED, Sickel AE, Trikha A, Levin A, Reynolds V: Axis I comorbidity of borderline personality disorder. Am J Psychiatry 1998, I55:1733-9.

\section{FlOOOPrime}

\section{RECOMMENDED}

5. Grant BF, Chou SP, Goldstein RB, Huang B, Stinson FS, Saha TD, Smith SM, Dawson DA, Pulay AJ, Pickering RP, Ruan WJ: Prevalence, correlates, disability, and comorbidity of DSM-IV borderline personality disorder: results from the Wave 2 National Epidemiologic Survey on Alcohol and Related Conditions. J Clin Psychiatry 2008, 69:533-45.

\section{FlOOOPrime
RECOMMENDED}

6. Zanarini MC, Frankenburg FR, Hennen J, Reich DB, Silk KR: Axis I comorbidity in patients with borderline personality disorder: 6-year follow-up and prediction of time to remission. Am J Psychiatry 2004, I 61:2108-14.

\section{FlOOOPrime}

\section{RECOMMENDED}

7. McGlashan TH, Grilo CM, Skodol AE, Gunderson JG, Shea MT, Morey LC, Zanarini MC, Stout RL: The Collaborative Longitudinal Personality Disorders Study: baseline Axis I/II and II/II diagnostic co-occurrence. Acta Psychiatr Scand 2000, 102:256-64

8. Banzhaf A, Ritter K, Merkl A, Schulte-Herbrüggen O, Lammers C, Roepke S: Gender differences in a clinical sample of patients with borderline personality disorder. J Pers Disord 2012, 26:368-80.

\section{FlOOOPrime}

9. Silverman $\mathrm{MH}$, Frankenburg FR, Reich DB, Fitzmaurice G, Zanarini MC: The course of anxiety disorders other than PTSD in patients with borderline personality disorder and Axis II comparison subjects: a I0-year follow-up study. J Pers Disord 2012, 26:804-14.
10. Zanarini MC, Hörz S, Frankenburg FR, Weingeroff J, Reich DB, Fitzmaurice G: The 10-year course of PTSD in borderline patients and axis II comparison subjects. Acta Psychiatr Scand 20II, I24:349-56.

\section{FIOOOPRIM
RECOMMENDED}

II. Nepon J, Belik S, Bolton J, Sareen J: The relationship between anxiety disorders and suicide attempts: findings from the National Epidemiologic Survey on Alcohol and Related Conditions. Depress Anxiety 2010, 27:791-8.

12. Cougle JR, Keough ME, Riccardi CJ, Sachs-Ericsson N: Anxiety disorders and suicidality in the National Comorbidity SurveyReplication. J Psychiatr Res 2009, 43:825-9.

13. Ozkan M, Altindag A: Comorbid personality disorders in subjects with panic disorder: do personality disorders increase clinical severity? Compr Psychiatry 2005, 46:20-6.

14. Friedman S, Jones JC, Chernen L, Barlow DH: Suicidal ideation and suicide attempts among patients with panic disorder: a survey of two outpatient clinics. Am J Psychiatry 1992, 149:680-5.

15. McKay D, Kulchycky S, Danyko S: Borderline personality and obsessive-compulsive symptoms. J Pers Disord 2000, I4:57-63.

16. Harned MS, Rizvi SL, Linehan MM: Impact of co-occurring posttraumatic stress disorder on suicidal women with borderline personality disorder. Am J Psychiatry 2010, 167:1210-7.

\section{FlOOOPrime}

\section{RECOMMENDED}

17. Rüsch N, Corrigan PW, Bohus M, Kühler T, Jacob GA, Lieb K: The impact of posttraumatic stress disorder on dysfunctional implicit and explicit emotions among women with borderline personality disorder. J Nerv Ment Dis 2007, I95:537-9.

18. Pagura J, Stein MB, Bolton JM, Cox BJ, Grant B, Sareen J: Comorbidity of borderline personality disorder and posttraumatic stress disorder in the U.S. population. J Psychiatr Res 2010, 44:1 190-8.

19. Bateman A, Fonagy P: Effectiveness of partial hospitalization in the treatment of borderline personality disorder: a randomized controlled trial. Am J Psychiatry 1999, I56:1563-9.

20. Bateman A, Fonagy P: Treatment of borderline personality disorder with psychoanalytically oriented partial hospitalization: an I 8-month follow-up. Am J Psychiatry 200I, I 58:36-42.

21. Bellino S, Rinaldi C, Bogetto F: Adaptation of interpersonal psychotherapy to borderline personality disorder: a comparison of combined therapy and single pharmacotherapy. Can J Psychiatry 2010, 55:74-8I.

22. Bellino S, Zizza M, Rinaldi C, Bogetto F: Combined therapy of major depression with concomitant borderline personality disorder: comparison of interpersonal and cognitive psychotherapy. Can J Psychiatry 2007, 52:718-25.

23. Bellino S, Zizza M, Rinaldi C, Bogetto F: Combined treatment of major depression in patients with borderline personality disorder: a comparison with pharmacotherapy. Can J Psychiatry 2006, 5 I:453-60.

24. Clarkin JF, Levy KN, Lenzenweger MF, Kernberg OF: Evaluating three treatments for borderline personality disorder: a multiwave study. Am J Psychiatry 2007, 164:922-8.

25. Doering S, Hörz S, Rentrop M, Fischer-Kern M, Schuster P, Benecke C, Buchheim A, Martius P, Buchheim P: Transference-focused psychotherapy $v$. treatment by community psychotherapists for borderline personality disorder: randomised controlled trial. $\mathrm{Br} J$ Psychiatry 2010, 196:389-95.

26. Gratz KL, Gunderson JG: Preliminary data on an acceptancebased emotion regulation group intervention for deliberate self-harm among women with borderline personality disorder. Behav Ther 2006, 37:25-35.

\section{FlOOOPrime}

RECOMMENDED

27. Soler J, Pascual JC, Tiana T, Cebrià A, Barrachina J, Campins MJ, Gich I, Alvarez E, Pérez V: Dialectical behaviour therapy skills training compared to standard group therapy in borderline 
personality disorder: a 3-month randomised controlled clinical trial. Behav Res Ther 2009, 47:353-8.

28. Turner RM: Naturalistic Evaluation of Dialectical Behavioral Therapy-Oriented Treatment for Borderline Personality Disorder. Cog Behav Prac 2000, 7:4I3-4I9.

29. Koons CR, Robins CJ, Tweed JL, Lynch TR, Gonzalez AM, Morse JQ, Bishop GK, Butterfield MI, Bastian LA: Efficacy of dialectical behavior therapy in women veterans with borderline personality disorder. Behavior Therapy 200I, 32:37I-390.

30. Davidson K, Norrie J, Tyrer P, Gumley A, Tata P, Murray H, Palmer S: The effectiveness of cognitive behavior therapy for borderline personality disorder: results from the borderline personality disorder study of cognitive therapy (BOSCOT) trial. J Pers Disord 2006, 20:450-65.

31. Davidson KM, Tyrer P, Norrie J, Palmer SJ, Tyrer H: Cognitive therapy v. usual treatment for borderline personality disorder: prospective 6-year follow-up. Br J Psychiatry 2010, 197:456-62.

\section{FIOOOPrime}

\section{RECOMMENDED}

32. Linehan MM, Heard HL, Armstrong HE: Naturalistic follow-up of a behavioral treatment for chronically parasuicidal borderline patients. Arch Gen Psychiatry 1993, 50:971-4.

33. Cottraux J, Note ID, Boutitie F, Milliery M, Genouihlac V, Yao SN, Note B, Mollard E, Bonasse F, Gaillard S, Djamoussian D, Guillard CdM, Culem A, Gueyffier F: Cognitive therapy versus Rogerian supportive therapy in borderline personality disorder. Two-year followup of a controlled pilot study. Psychother Psychosom 2009, 78:307-I6.

34. Stoffers JM, Völlm BA, Rücker G, Timmer A, Huband N, Lieb K: Psychological therapies for people with borderline personality disorder. Cochrane Database Syst Rev 2012, 8:CD005652.

\section{FlOOOPrime \\ RECOMMENDED}

35. Bradley R, Greene J, Russ E, Dutra L, Westen D: A multidimensional meta-analysis of psychotherapy for PTSD. Am J Psychiatry 2005, 162:214-27.

36. Westen $D$, Morrison K: A multidimensional meta-analysis of treatments for depression, panic, and generalized anxiety disorder: an empirical examination of the status of empirically supported therapies. J Consult Clin Psychol 200I, 69:875-99.

37. Feeny NC, Zoellner LA, Foa EB: Treatment outcome for chronic PTSD among female assault victims with borderline personality characteristics: a preliminary examination. J Pers Disord 2002, 1 6:30-40.

\section{FlOOOPrime}

\section{RECOMMENDED}

38. Clarke SB, Rizvi SL, Resick PA: Borderline personality characteristics and treatment outcome in cognitive-behavioral treatments for PTSD in female rape victims. Behav Ther 2008, 39:72-8.

\section{FlOOOPrime \\ FIOOPYIme}

39. Mueser KT, Rosenberg SD, Xie H, Jankowski MK, Bolton EE, Lu W, Hamblen JL, Rosenberg HJ, McHugo GJ, Wolfe R: A randomized controlled trial of cognitive-behavioral treatment for posttraumatic stress disorder in severe mental illness. J Consult Clin Psychol 2008, 76:259-7I.

40. Dreessen L, Arntz A: The impact of personality disorders on treatment outcome of anxiety disorders: best-evidence synthesis. Behav Res Ther 1998, 36:483-504.

4I. Mennin DS, Heimberg RG: The impact of comorbid mood and personality disorders in the cognitive-behavioral treatment of panic disorder. Clin Psychol Rev 2000, 20:339-57.

42. Harned M, Linehan M: Integrating Dialectical Behavior Therapy and Prolonged Exposure to treat co-occurring borderline personality disorder and PTSD: Two case studies. Cog Behav Prac 2008, I 5:263-276.

43. Harned MS: Treatment of posttraumatic stress disorder with comorbid borderline personality disorder. In Handbook of treating variants and complications in anxiety disorders. Edited by McKay D, Storch E. New York, NY: Springer Press; 2013.

44. Harned MS, Korslund KE, Foa EB, Linehan MM: Treating PTSD in suicidal and self-injuring women with borderline personality disorder: development and preliminary evaluation of a Dialectical Behavior Therapy Prolonged Exposure Protocol. Behav Res Ther 2012, 50:381-6.

\section{FlOOOPrime}

\section{RECOMMENDED}

45. Pabst A, Schauer M, Bernhardt K, Ruf M, Goder R, Rosentraeger R, Elbert T, Aldenhoff J, Seeck-Hirschner M: Treatment of patients with borderline personality disorder and comorbid posttraumatic stress disorder using narrative exposure therapy: a feasibility study. Psychother Psychosom 2012, 81:6I-3.

\section{FIOOOPrime
RECOMMENDED}

46. Bohus M, Dyer AS, Priebe K, Krüger A, Steil R: Dialektisch Behaviorale Therapie für Posttraumatische Belastungsstörung nach sexualisierter Gewalt in der Kindheit und Jugend (DBTPTSD). Psychother Psychosom Med Psychol 201 I, 6 I:I40-7.

47. Steil R, Dyer A, Priebe K, Kleindienst N, Bohus M: Dialectical behavior therapy for posttraumatic stress disorder related to childhood sexual abuse: a pilot study of an intensive residential treatment program. J Trauma Stress 201 I, 24:102-6.

\section{FlOOOPrime} RECOMMENDED

48. Bohus M, Kruger A, Dyer A, Priebe K, Steil R: Residential DBT program for patients with borderline personality disorder and PTSD after childhood sexual abuse: A controlled randomized trial. Presented at the 8th Annual NIMH Conference of the National Education Alliance for Borderline Personality Disorder. 20I I; Seattle, WA.

49. Cloitre M, Stovall-McClough KC, Nooner K, Zorbas P, Cherry S, Jackson CL, Gan W, Petkova E: Treatment for PTSD related to childhood abuse: a randomized controlled trial. Am J Psychiatry 2010, 167:915-24.

\section{FlOOOPrime}

\section{RECOMMENDED}

50. Wilamowska ZA, Thompson-Hollands J, Fairholme CP, Ellard KK Farchione TJ, Barlow DH: Conceptual background, development, and preliminary data from the unified protocol for transdiagnostic treatment of emotional disorders. Depress Anxiety 2010, 27:882-90.

5I. Eaton NR, Krueger RF, Keyes KM, Skodol AE, Markon KE, Grant BF, Hasin DS: Borderline personality disorder co-morbidity: relationship to the internalizing-externalizing structure of common mental disorders. Psychol Med 20II, 4I: I04I-50.

\section{FIOOOPrime
RECOMMENDED}

52. Miller MW, Wolf EJ, Reardon A, Greene A, Ofrat S, Mclnerney S: Personality and the latent structure of PTSD comorbidity. J Anxiety Disord 2012, 26:599-607.

\section{FlOOOPrime}

53. Gratz KL, Tull MT, Gunderson JG: Preliminary data on the relationship between anxiety sensitivity and borderline personality disorder: the role of experiential avoidance. J Psychiatr Res 2008, 42:550-9.
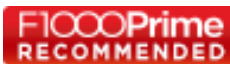